\title{
DESARROLLO CONCEPTUAL DE LA EDUCACIÓN AMBIENTAL EN EL CONTEXTO COLOMBIANO ${ }^{1}$
}

\author{
Luisa S. Paz M. ${ }^{2}$ \\ William R. Avendaño C. ${ }^{3}$ \\ Abad E. Parada-Trujillo ${ }^{4}$ \\ Recibido el 1 de septiembre de 2013 y aprobado el 28 de mayo de 2014, , \\ actualizado el 13 junio de 2014
}

\section{RESUMEN}

La Educación Ambiental (EA) ha tenido un auge significativo, se trata de una respuesta hacia la necesidad de un cambio social estructural. Sus fundamentos se encuentran tanto en las normas internacionales como locales, y su desarrollo conceptual ha sido amplio y complejo. El objetivo del presente artículo es explorar algunos elementos normativos y conceptuales que fundamentan la EA, así como la adopción de la misma en términos curriculares en Colombia. El método empleado se circunscribe en el análisis documental, razón por la que se abordó una amplia información de la cual se realizaron inferencias de acuerdo al propósito del artículo. Dentro de los resultados, se encontró que la EA ha sido asumida conforme a las características de cada periodo histórico, en especial, del siglo inmediatamente pasado con un gran cúmulo de normas internacionales y nacionales, y que su implementación desde la escuela debe corresponder a una reflexión conjunta y colectiva de todos los actores escolares, tanto en su diseño como implementación. Cualquier enfoque de la educación ambiental resulta válido siempre que corresponda a los objetivos trazados por la escuela en coherencia con las necesidades y problemáticas locales, aquellas de las que son parte los educandos. Se concluye que las normas internacionales y locales en materia de educación ambiental son evidencia de la trascendencia que tiene la protección del medio ambiente y que los enfoques desarrollados desde el terreno conceptual posibiliten el cambio siempre que se integren elementos tanto críticos como cognitivos o socio-cognitivos.

\section{PALABRAS CLAVE}

Educación ambiental, desarrollo conceptual, contexto colombiano.

\section{CONCEPTUAL DEVELOPMENT OF ENVIRONMENTAL EDUCATION IN THE COLOMBIAN CONTEXT}

\begin{abstract}
Environmental Education (EE) has had a significant growth as a response to the need for structural social change. Its foundations are found in both international and local standards, and its conceptual development has been extensive and complex. The aim of this article is to explore some statutory and conceptual elements underpinning EE, and its adoption in curricular terms in Colombia. The method used is limited to documentary analysis reason why a wide range of information was addressed from which inferences were made according to the purpose of the article. Among the results, it was found that EE has been taken according to the characteristics of each historical period, particularly since the 70 's in the twentieth century with a wealth of international and national standards, and that its implementation from school should respond to a joint and collective reflection of all school actors, both in its design and implementation. Any
\end{abstract}


environmental education approach is valid whenever appropriate to the goals set by the school in line with local needs and problems, which are part of the learners' reality. It is concluded that international and local environmental education norms are evidence of the importance that environmental protection and the approaches developed from the conceptual change make change possible provided that they integrate both critical and cognitive or socio-cognitive elements.

\section{KEY WORDS}

Environmental education, conceptual development, Colombian context.

\section{INTRODUCCIÓN}

En Colombia, la Educación Ambiental (EA) que se imparte en las instituciones escolares se encuentra instituida por el artículo 14 literal c) de la Ley 115 de 1994. La norma expresa que es obligatorio en los niveles de educación formal (preescolar, básica y media) impartir la "enseñanza de la protección del ambiente, la ecología y la preservación de los recursos naturales", de conformidad con la Carta Política de 1991. Esta norma es de vital importancia en el marco de los acuerdos y los compromisos suscritos por el Estado colombiano en procura de alcanzar un desarrollo sostenible. La EA compromete a todos los individuos, las familias, la sociedad en general y los poderes públicos del Estado (Valdés, 2000, p. 623), porque la humanidad se encuentra con una crisis ambiental (Orellana, 2002, p. 221) la cual corresponde a la "crisis de nuestro tiempo" y que "no es una catástrofe ecológica resultante de la evolución de la naturaleza, sino producida por el pensamiento con el que hemos construido y destruido nuestro mundo" (Leff, 2000, p. 67). En otras palabras, hoy más que nunca es perentorio el diseño e implementación de acciones por parte del Estado que implique la participación de la sociedad y, en especial, la escuela como espacio para la reproducción de la cultura y transformación social.

Ya desde hace dos décadas, no solo Colombia sino los Estados en general, han reconocido la urgencia de realizar acciones concretas y efectivas para promover el desarrollo sostenible, es decir, hacer uso de los recursos sin poner en peligro la existencia de las generaciones futuras. Un ejemplo de ello es la inclusión de aspectos medioambientales en las constituciones políticas de los diferentes Estados, y en América Latina "la mayoría de los países hicieron cambios [...], en los que hubo un mayoritario reconocimiento constitucional a la materia ambiental, tema que, salvo excepciones, no había aparecido, hasta el momento, de manera tan notoria en las constituciones" (Acuña, 2010, p. 113). Esto es relevante desde el punto de vista político, pues se entiende que la inclusión de estas normas ambientales a nivel constitucional es debido a la importancia, urgencia y al consenso de los pueblos por mejorar el estado de crisis en materia ambiental. Las cartas políticas contienen los principios fundamentales para la creación y organización de los poderes públicos además de las normas generales que enmarcan la totalidad del ordenamiento jurídico, así como las garantías individuales y sociales "que deben ser respetados por la totalidad del sistema jurídico" (Vázquez, 1975, p. 589). Entonces, los Estados que referencian normas medioambientales desde la misma Constitución, admiten la superioridad de la misma, razón por la que debe ser desarrollada y garantizada por el resto del sistema jurídico. En el Cuadro 1 se hace una síntesis de algunas normas constitucionales en América Latina que han incluido los países en sus cartas 
políticas. En algunas de ellas se ha realizado la inclusión de normas medioambientales con capítulos completos, otras en las secciones de derechos y garantías de las personas.

Cuadro 1. Algunas normas constitucionales en América Latina referidas a la protección del medio ambiente

\begin{tabular}{|c|c|}
\hline $\begin{array}{c}\text { Constitución } \\
\text { Política }\end{array}$ & Artículos que obligan a la protección del medio ambiente \\
\hline Argentina & $\begin{array}{l}\text { Artículo 41- Todos los habitantes gozan del derecho a un ambiente sano, } \\
\text { equilibrado, apto para el desarrollo humano y para que las actividades } \\
\text { productivas satisfagan las necesidades presentes sin comprometer las de } \\
\text { las generaciones futuras, y tienen el deber de preservarlo. El daño } \\
\text { ambiental generará prioritariamente la obligación de recomponer, según lo } \\
\text { establezca la ley [...]. }\end{array}$ \\
\hline Brasil & $\begin{array}{l}\text { Artículo 225. Todos tienen derecho a un medio ambiente ecológicamente } \\
\text { equilibrado, bien de uso común del pueblo y esencial para una sana } \\
\text { calidad de vida, imponiéndose al Poder Público y a la colectividad el deber } \\
\text { de defenderlo y preservarlo para las generaciones presentes y futuras [...]. }\end{array}$ \\
\hline Chile & $\begin{array}{l}\text { Artículo 19. La Constitución asegura a todas las personas: } \\
\text { Núm. } 8^{\circ} \text { : El derecho a vivir en un medio ambiente libre de contaminación. } \\
\text { Es deber del Estado velar para que este derecho no sea afectado y tutelar } \\
\text { la preservación de la naturaleza [...]. }\end{array}$ \\
\hline Colombia & $\begin{array}{l}\text { Artículo 79. Todas las personas tienen derecho a gozar de un ambiente } \\
\text { sano. La ley garantizará la participación de la comunidad en las decisiones } \\
\text { que puedan afectarlo. Es deber del Estado proteger la diversidad e } \\
\text { integridad del ambiente, conservar las áreas de especial importancia } \\
\text { ecológica y fomentar la educación para el logro de estos fines. } \\
\text { Artículo } 80 \text {. El Estado planificará el manejo y aprovechamiento de los } \\
\text { recursos naturales, para garantizar su desarrollo sostenible, su } \\
\text { conservación, restauración o sustitución [...]. }\end{array}$ \\
\hline México & $\begin{array}{l}\text { Artículo } 4^{\circ} \text {. Parágrafo } 5^{\circ} \text {. Toda persona tiene derecho a un medio } \\
\text { ambiente sano para su desarrollo y bienestar. El Estado garantizará el } \\
\text { respeto a este derecho. El daño y deterioro ambiental generáá a } \\
\text { responsabilidad para quien lo provoque en términos de lo dispuesto por la } \\
\text { ley. }\end{array}$ \\
\hline
\end{tabular}




\begin{tabular}{|c|c|}
\hline Panamá & $\begin{array}{l}\text { Artículo 114. Es deber fundamental del Estado garantizar que la } \\
\text { población viva en un ambiente sano y libre de contaminación [...]. } \\
\text { Articulo } 115 \text {. El Estado y todos los habitantes del territorio nacional tienen } \\
\text { el deber de propiciar un desarrollo social y económico que prevenga la } \\
\text { contaminación del ambiente, mantenga el equilibrio ecológico y evite la } \\
\text { destrucción de los ecosistemas. } \\
\text { Articulo } 116 \text {. El Estado reglamentará, fiscalizará y aplicará oportunamente } \\
\text { las medidas necesarias para garantizar que la utilización y el } \\
\text { aprovechamiento de la fauna terrestre, fluvial y marina, así como de los } \\
\text { bosques, tierras y aguas, se lleven a cabo racionalmente, de manera que } \\
\text { se evite su depredación y se asegure su preservación, renovación y } \\
\text { permanencia. } \\
\text { Articulo } 117 \text {. La Ley reglamentará el aprovechamiento de los recursos } \\
\text { naturales no renovables [...]. }\end{array}$ \\
\hline Paraguay & $\begin{array}{l}\text { Artículo } 7^{\circ} \text {. Toda persona tiene derecho a habitar en un ambiente } \\
\text { saludable y ecológicamente equilibrado. Constituyen objetivos prioritarios } \\
\text { de interés social la preservación, la conservación, la recomposición y el } \\
\text { mejoramiento del ambiente, así como su conciliación con el desarrollo } \\
\text { humano integral. Estos propósitos orientarán la legislación y la política } \\
\text { gubernamental pertinente. } \\
\text { Artículo } 8^{\circ} \text {. Las actividades susceptibles de producir alteración ambiental } \\
\text { serán reguladas por la ley. Asimismo, ésta podrá restringir o prohibir } \\
\text { aquellas que califique peligrosas [...]. }\end{array}$ \\
\hline Perú & 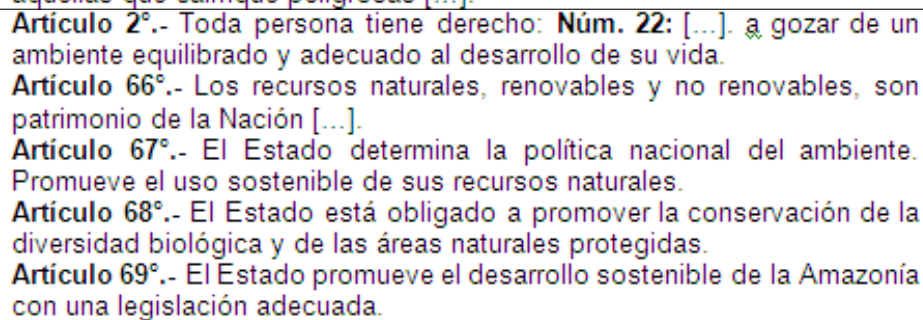 \\
\hline Venezuela & $\begin{array}{l}\text { Artículo 127. Es un derecho y un deber de cada generación proteger y } \\
\text { mantener el ambiente en beneficio de sí misma y del mundo futuro [...]. El } \\
\text { Estado protegerá el ambiente, la diversidad biológica, genética, los } \\
\text { procesos ecológicos, los parques nacionales y monumentos naturales [...]. } \\
\text { Es una obligación fundamental del Estado, con la activa participación de la } \\
\text { sociedad, garantizar que la población se desenvuelva en un ambiente libre } \\
\text { de contaminación [...]. }\end{array}$ \\
\hline
\end{tabular}
Panamá, Paraguay, Perú y Venezuela.

Los Estados latinoamericanos han instituido la protección del medio ambiente a partir de las constituciones políticas de cada país. Como se puede observar en el Cuadro 1, el fin de todos es similar: trabajar en pro de una cultura que fomente la preservación de los hábitats, la biodiversidad y los recursos naturales renovables y no renovables, brindar una mejor calidad de vida a sus habitantes en ambientes libres de contaminación. Estas normas de nivel constitucional sugieren que dichos contenidos deben ser asumidos desde una política de Estado, pues tienen relación directa con el marco axiológico de cada Nación. Al mismo tiempo, aparece de forma homogénea el medio ambiente como un derecho, que implica un conjunto de obligaciones reciprocas, es decir, toda persona tiene derecho a un ambiente sano pero a su vez todo individuo tiene la obligación de propiciar un respeto por el medio ambiente. Cada una de las normas referenciadas en el Cuadro 1, identifican a la persona como sujeto activo del derecho, pero a su vez es sujeto pasivo de esta relación.

Los avances en el derecho constitucional impulsaron en los Estados una revisión y reforma de las cartas políticas en coherencia con las discusiones medioambientales. Las transformaciones comenzaron en las década de los setenta, específicamente en 1972, "en consonancia con el espacio que comenzaban a ocupar, en todo el mundo, las ideas sobre la protección del medio ambiente y el desarrollo sostenible" (Brañes, 2001, p. 47). El concepto de desarrollo sostenible está implícitamente asociado con el medio ambiente, y ello implica la necesidad del uso racional de los recursos a fin de no afectar la vida de 
las generaciones presentes y futuras. La materialización de nuevos valores estatales y objetivos gubernamentales, se ha dado a partir de la aprobación y ratificación de instrumentos internacionales como la Declaración de Río (1992) o Declaración Salónica (1997). La Declaración de Río de 1992 y la firma de Colombia como Estado parte de esta manifestación es una "evidencia del compromiso adquirido por el gobierno [...] en el desarrollo de actividades que garanticen de forma tangible la protección del medio ambiente y el desarrollo sostenible" (Avendaño, 2011, p. 48).

De este modo, la EA y las prácticas que sobre ella se tejen, desde el punto de vista formativo y político, son instrumentos necesarios para afrontar los problemas del medio ambiente, los cuales se han agravado e intensificado en las últimas décadas (Casares, 2000, p. 43). Al reconocer la educación como un proceso de reproducción cultural (Durkheim, 1976, p. 17; Giroux, 1986, p. 64; Avendaño \& Parada, 2011, p. 400; Ball, 2001, p. 6; Dewey, 2004, p. 15) en donde los sujetos de aprendizaje pueden a partir de la formación que se les brinda hacer cambios significativos en sus entornos, entonces se debe reconocer la EA como un medio efectivo para construir nuevos modelos de pensamiento, mejorar la calidad de vida de los hombres y contribuir al aseguramiento de la supervivencia de todas las especies en la tierra. En fin, la educación permite un cambio de comportamiento de los sujetos de aprendizaje lo cual se traduce en transformaciones a nivel de pensamiento, sentimientos y acciones (Tyler, 1986, p. 13).

Sin embargo, en la práctica la EA presenta dificultades al igual que toda la educación en general pues esta se encuentra marcada por viejas formas escolarizadas que poco responden a las características y exigencias de la sociedad del conocimiento (Pilonieta, 2010; Avendaño \& Parada, 2011, p. 401). Por otro lado, las propuestas en las que se consignan la enseñanza de la EA requieren de una mayor "reflexión que ayude a integrar la educación ambiental como proyecto institucional, con las propuestas de aula, las cuales no tienen suficientemente en cuenta los verdaderos principios y fundamentos conceptuales de la educación ambiental" (Pardo, 1995, p. 9). En otras palabras, existe una urgencia por definir verdaderos programas de EA a nivel institucional, de tal suerte que puedan desde la interdisciplinariedad ser abordados para que esta formación resulte significativa desde la misma aula o cualquier lugar escolar donde se da el proceso de formación. Pero para ello, es importante hacer identificar y analizar los elementos jurídicos que fundamentan la educación ambiental, además de los aspectos conceptuales desarrollados en torno al tema, a fin de hacer algunas recomendaciones de tipo pedagógico.

El presente artículo de revisión explora la noción conceptual de la educación ambiental, algunos elementos normativos de orden internacional que muestran la evolución del objeto de estudio, el marco normativo nacional que fundamenta el necesario cambio social, político y educativo en materia ambiental, la implementación de la educación ambiental desde el terreno curricular en Colombia y las construcciones que se han realizado en el campo de la educación ambiental en términos y que atienden a concebir las relaciones entre el hombre y el medio ambiente.

\section{EDUCACIÓN AMBIENTAL (EA): ACERCAMIENTO CONCEPTUAL}

Intentar ofrecer un concepto de la educación ambiental, es una meta compleja que requiere de un análisis de los diversos elementos que componen este 
constructo. Por un lado, es necesario hacer un acercamiento al concepto de educación y de medio ambiente de forma separada a fin de ofrecer una construcción conceptual válida. La educación desde su concepción epistemológica tiene su origen en dos términos latinos: educere y es-ducere. El primero de ello hace mención a los verbos nutrir o alimentar, y el segundo a una acción de llevar o sacar de adentro hacia afuera (Pérez, 2003, p. 119). Por lo tanto, desde esta base se comprende que la educación es un proceso ejercido, primeramente, desde el exterior o por agentes externos, y luego como una acción autónoma e individual. Ahora bien, por educación se debe entender que se trata de un proceso de reproducción y transformación cultural, entendida la cultura como todas aquellas construcciones colectivas que componen los saberes, las creencias, las conductas y la organización social. De allí que hable en la actualidad de la pluriculturalidad y de la necesidad de una educación pluricultural, es decir, de procesos en las escuelas basados en la reproducción y transformación de los saberes, las creencias, la cultura, las conductas y la organización social. Asimismo, dependiendo del contexto de las escuelas, la educación tendrá un rol o función específica respecto de aquello en lo que se debe educar.

En cuanto al término medio ambiente, autores como Gómez (2002, p. 37), Fernández \& Leiva (2003), Fernández, Moreno, De la Losa \& Calaforra (2005, p. 1058) y otros, conciben el medio ambiente como la interrelación de los elementos físicos, biológicos, económicos, sociales, culturales y estéticos, que pueden afectar de un modo u otro la vida en la tierra de los seres presentes y las de las futuras generaciones. Así, la EA se entenderá como un proceso de reproducción y transformación cultural, es decir, los saberes, las conductas, las creencias y demás, en torno al cuidado y uso racional de cada uno de esos elementos que integran el medio ambiente, para no arriesgar las condiciones requeridas para una vida digna.

Para Nóvoa (2009, p. 198) la EA es una vía para el "replanteamiento de nuestras relaciones con la biosfera, a la vez que un instrumento de transformación social y empoderamiento de los más débiles, todo ello con la meta final de conseguir sociedades más armónicas y equitativas". De este modo, una de las funciones esenciales de la EA es abrir espacios de reflexión en torno a las relaciones del hombre respecto de los otros elementos que conforman el medio ambiente, y a su vez se traduce en acciones concretas para mejorar la calidad de vida de las personas, especialmente las que se encuentran en situaciones de vulnerabilidad o excluidas.

\section{EVOLUCIÓN DE LA EDUCACIÓN AMBIENTAL DESDE EL CARÁCTER NORMATIVO INTERNACIONAL}

Si bien los avances significativos en materia de EA se dieron en los años setenta, ya para finales del siglo XX los temas de protección del medio ambiente y educación se hacían visiblemente necesarios. El primero se puede ubicar en 1968 en París con la Conferencia de la Biosfera en donde "se dan pautas para incorporar la EA al sistema educativo" (Moreno, 2008). Pese a este tipo de referencias sobre el concepto de Educación Ambiental, el reconocimiento formal de su importancia se establece en la Conferencia de las Naciones Unidas sobre el Medio Ambiente Humano en Estocolmo los días 5-16 de junio de 1972, llamada Declaración de Estocolmo. En ella se resaltan dos elementos: una proclamación y un conjunto de principios alrededor del tema del medio ambiente. En la proclamación resulta relevante que: a) los Estados miembros admiten que 
el avance de la ciencia y la tecnología pueden influir, en gran escala, sobre el medio ambiente; b) la protección del medio humano es fundamental para el bienestar y el desarrollo de los pueblos; c) el hombre debe reflexionar permanentemente en sus descubrimientos e invenciones a fin de no causar daños incalculables a la humanidad ni al medio; d) hay un deterioro evidente en regiones de la tierra, existe una grave contaminación del agua, el aire, la tierra y los seres vivos, se está gestando un desequilibrio ecológico y se agotan los recursos de la tierra; e) los niveles de vida de muchos seres humanos son muy bajos, presentándose una problemática alimentaria, de vivienda, educación, sanidad, higiene, etc.

El segundo aspecto relevante de la Declaración de Estocolmo, es la definición de 24 principios entre los que se destacan: a) derechos fundamentales (libertad, igualdad y disfrute de condiciones de vida adecuadas); b) preservación de los recursos naturales para el goce de generaciones presentes y futuras; c) mantenerse, restaurarse o mejorarse la capacidad de la tierra para producir recursos vitales para el hombre; d) responsabilidad del hombre en la preservación de la flora y fauna; e) uso adecuado de los recursos no renovables; f) poner fin a descargas de sustancias tóxicas y otras que causen daño a los ecosistemas; g) obligación de los Estados en impedir la contaminación de los mares; etc. También en la década de los setenta y ochenta se dan algunas otras citas para el abordaje de los temas de educación relacionados con aspectos medioambientales y ecológicos como el Coloquio Internacional sobre Educación relativa al Medio Ambiente (Belgrado, 1975), la Conferencia Intergubernamental sobre Educación relativa al Medio Ambiente (Tbilisi, 1977), , el Congreso Internacional de Educación y Formación sobre Medio Ambiente (Moscú, 1987).

Ya en 1992 con la Cumbre de la Tierra o Conferencia de las Naciones Unidas sobre el Medio Ambiente y el Desarrollo (CNUMAD), 172 gobiernos aprobaron acuerdos relacionados con el desarrollo sostenible. Un producto de este encuentro fue la Declaración de Río sobre el Medio Ambiente conformada por 27 principios, destacándose el derecho de las personas a una vida saludable, el derecho de los Estados a aprovechar sus recursos y el deber de velar por las actividades que se realizan en su jurisdicción, equivalencia entre desarrollo y necesidades sin arriesgar las generaciones presente y futuras, integración de la protección del medio ambiente en la agenda de desarrollo sostenible, la cooperación y solidaridad mundial en función de conservar, proteger y restablecer la salud e integridad del ecosistema de la Tierra, etc. (Avendaño, 2011, p. 48). En los años noventa el concepto de desarrollo sostenible asociado al marco económico señala un nuevo enfoque en los temas ambientales, ecológicos y educativos.

\section{EL DESARROLLO NORMATIVO EN COLOMBIA}

En el contexto colombiano, las políticas y acciones en materia de EA asumieron el enfoque normativo con el Plan Nacional de Desarrollo Ambiental titulado El salto social, hacia el desarrollo humano sostenible en el año 1997. Variantes como la pobreza, la violencia y el medio ambiente fueron asumidos en el plan, los cuales constituyen elementos muy acordes con la realidad de la Nación y las necesidades de las comunidades. Si se observa, esta perspectiva corresponde a un enfoque sistémico, es decir, asumir la EA como un conjunto de elementos interrelacionados en los que es necesario armonizarlos dentro de la totalidad del sistema porque la falla de uno afecta a los demás. El Plan de Desarrollo Ambiental de 1997 precisó: 
[...] para obtener un nuevo ciudadano, se requiere de la construcción de un proceso de sensibilización, concientización y participación y propende porque mediante la educación el hombre mejore su actuación sobre la naturaleza, haciendo un aprovechamiento sostenible de los recursos de que dispone y a los cuales tiene acceso. (Ministerio del Medio Ambiente \& Ministerio de Educación Nacional, 2002, p. 7)

Ya para el año de 1998 y 2002 la Política Ambiental del Plan de Desarrollo se diseña en virtud de un Cambio para construir la paz, y este proyecto privilegia "los instrumentos y acciones dirigidos a fomentar entre los ciudadanos la ética, la responsabilidad, el conocimiento y la capacidad para prever y enfrentar colectivamente, la solución de los problemas ambientales" (Ministerio del Medio Ambiente \& Ministerio de Educación Nacional, 2002, p. 7). Lo que se logra observar de estas dos políticas (El salto social, hacia el desarrollo humano sostenible y Cambio para construir la paz) es la incapacidad de dar continuidad a las acciones de un gobierno a otro. En dicho sentido, es de conocimiento que ninguna política pública, y sobre todo las asociadas a la construcción de ciudadanía, valores y conciencia, como lo es la EA, puede generar efectos en corto o mediano plazo. Por lo tanto, la política que se desarrolla debe ser trabajada a lo largo del tiempo para que se alcancen resultados significativos en dicho sentido, aún más si se considera el enfoque sistémico dado a la EA.

Anteriores a estas políticas, ya la Ley 115 de 1994 (Ley General de Educación) en su artículo $5^{\circ}$ establecía como uno de los fines de la educación "la adquisición de una conciencia para la conservación, protección y mejoramiento del medio ambiente, de la calidad de vida, del uso racional de los recursos naturales [...]" y a su vez se expedía en el mismo año, el Decreto 1743 de 1994 por el cual se instituía el Proyecto de Educación Ambiental para todos los niveles de educación formal, con participación del Ministerio de Educación Nacional y el Ministerio de Ambiente. Resalta de este decreto, la obligación para las instituciones educativas de incluir dentro del Proyecto Educativo Institucional (PEI), "proyectos ambientales, escolares en el marco de diagnósticos ambientales, locales, regionales y/o nacionales, con miras a coadyuvar a la resolución de problemas ambientales específicos" (art. $\left.1^{\circ}\right)$. Sin embargo, los avances en este sentido han sido muy escasos, no se ha logrado establecer una verdadera articulación entre las instituciones públicas y los programas y acciones han correspondido a las directrices del gobierno de turno.

El marco referido a los proyectos escolares de tipo ambiental es relevante, porque su diseño debe responder a diagnósticos ambientales que incluya las problemáticas, los fenómenos, las necesidades y la relación del hombre con el medio ambiente. La pregunta en dicho sentido resalta: ¿han cumplido las instituciones educativas el diagnostico previo para el diseño de los proyectos ambientales en coherencia con el PEI de cada plantel? Y es que es vital, pues como se ha sostenido, la EA debe responder a las necesidades del entorno de los educandos y a sus características y requerimientos personales y colectivos.

El mismo Decreto 1743 de 1994, en su art. $2^{\circ}$ establece los principios rectores de la EA, es decir, del Proyecto Ambiental Escolar (PRAE): la interculturalidad, la formación de valores, la regionalización, la interdisciplinariedad, la participación y formación para la democracia, la gestión y resolución de problemas. El diseño del mismo, corresponderá a la totalidad de miembros de la comunidad educativa a partir de los diversos órganos del gobierno escolar (art. $3^{\circ}$ ), es decir, su diseño e implementación debe comprometer a la comunidad educativa en general conforme a sus intereses y a la forma en que comprenden sus relaciones con la naturaleza. De allí que se establezca como parte de los instrumentos utilizados 
para el desarrollo de los PRAE, la asesoría y el apoyo institucional a través de directivas y actos administrativos que coordinen y controlen las acciones de ejecución de los proyectos (art. $4^{\circ}$ ); la formación de docentes (art. 50); la evaluación permanente (art. $\left.6^{\circ}\right)$; el servicio social obligatorio (art. $7^{\circ}$ ); entre otras.

El otro documento esencial para la comprensión de la EA en Colombia son los lineamientos curriculares para el área de Ciencias Naturales y Educación Ambiental de 1995, de igual forma, anterior a las políticas ya señaladas. En él se establecen los 5 logros y objetivos de la EA para Colombia: a) concientización; b) conocimiento; c) valores, actitudes y comportamientos; d) competencia; y e) participación. La concientización se refiere a un despertar de la conciencia por el medio ambiente y sus problemas. El conocimiento se enfoca en el desarrollo de experiencias que le permitan al sujeto apropiarse de un saber básico en temas medioambientales. Los valores, las actitudes y los comportamientos buscan la construcción de valores y una mayor participación de los sujetos en el mejoramiento y protección del ambiente. La competencia permite que los sujetos identifiquen, anticipen y resuelvan problemas relacionados con la temática. Finalmente, la participación busca la implicación dinámica de los sujetos en la solución de los problemas ambientales.

La EA en Colombia se enfoca en la concepción de lineamientos generales y abarcadores, pero solo desde el escenario local se puede profundizar en objetivos concretos y significativos para las escuelas. Por ello, los lineamientos curriculares señalan que los sujetos de aprendizaje frente a las problemáticas ambientales, deben "ejercitar la reflexión crítica respecto a comportamientos hombre-naturaleza-ciencia-tecnología-sociedad", y que puede ser abordada desde diversas corrientes de la EA. Los problemas ambientales no pueden ser evaluados desde la dimensión física o química, sino que estos tienen asociados factores naturales, sociales, económicos, políticos, culturales, epistémicos, filosóficos, entre otros, y por lo tanto, su tratamiento debe ser de la misma complejidad atendiendo a todos los elementos que puedan incidir en los programas ambientales en las escuelas.

Las normas internacionales relacionadas en el apartado dos y las nacionales relacionadas en el presente apartado, así como los compromisos adquiridos por el Estado, y por ende, por toda la población, en especial al sector educativo, conducen a replantar las prácticas que se desarrollan dentro de las aulas, así como el papel del PEI, el currículo y el plan de estudios implementado. La reproducción de formas escolarizadas basadas en los contenidos temáticos y la memorización de los mismos a espaldas de las realidades y las necesidades de las comunidades son una manifiesta contradicción a los compromisos adquiridos y a la construcción de mejores estados de vida para el hombre.

\section{CONCEPCIONES Y CORRIENTES DE LA EDUCACIÓN AMBIENTAL}

El medio ambiente es un entorno vital en donde se interrelacionan "elementos físicos, biológicos, económicos, sociales, culturales y estéticos" (Gómez, 2002, p. 37; Fernández \& Leiva, 2003; Fernández et al., 2005, p. 1058; Martínez, 2005, p. 85), y en este panorama se ven envueltos los individuos, las comunidades, la sociedad en general. El medio ambiente es todo un grupo de circunstancias que le permite al hombre construir un modelo de vida. Resalta, al analizar la definición que comparte un gran número de autores sobre medio ambiente, la complejidad que reviste el tema pues no es un asunto meramente físico ni 
biológico, sino también sociocultural, económico, filosófico, estético, axiológico, etc.

Complejos y diversos son los desafíos que las actuales sociedades enfrentan en aras de salvaguardar el medio ambiente y como lo expresa la Organización de las Naciones Unidas para la Educación, la Ciencia y la Tecnología (UNESCO) el nuevo milenio es una "transición simbólica, más que corte tajante de épocas, el nuevo milenio es ocasión de valorar rutas y asumir nuevos compromisos" (UNESCO, 1998, p. 2). Una amplia regulación normativa acompañada de un despertar de la conciencia colectiva sobre las actividades humanas ha resultado útil para proteger la naturaleza y los recursos en los últimos años; sin embargo, no ha sido suficiente tomando en cuenta el gran despliegue tecnológico, el uso desaforado de los recursos naturales y la expropiación indiscriminada de los ecosistemas. Esto hace parte de la perspectiva del hombre, que producto de la Segunda Revolución Industrial, veía los recursos como fuentes inagotables (Ministerio de Educación - Gobierno de la ciudad de Buenos Aires, 2009, p. 13) y que a través del despliegue y desarrollo de la ciencia se podía dominar la naturaleza, idea que ya se gestionaba desde la modernidad (Porto, 2000, p. 11).

Se ha exigido a los entes gubernamentales correspondientes en cada país, un desarrollo legislativo coherente con las necesidades del medio ambiente que logre tomar acciones correctivas y preventivas. En otras palabras, un marco jurídico que regule, controle, inspeccione y sancione, a aquellos que en el ejercicio de sus labores pueden afectar al entorno natural y sus preciados bienes. En este sentido, a educación adquiere un papel fundamental en este tópico. Hay que forjar sólidas bases educativas para que los niños, niñas y jóvenes que se encuentran en proceso de formación se apropien de esta problemática, tomen posturas activas respecto del tema y participen como actores sociales en la construcción de una nueva realidad. Pardo (1995, p. 17) analiza las esferas en que actúan los seres humanos: la sociosfera, la tecnosfera y la biosfera. Estas se encuentran relacionadas entre sí: la sociosfera afecta la tecnosfera y la biosfera directamente, aunque también puede afectarla a través de la tecnosfera (Gráfico 1). De allí que describa la necesidad de hacer cambios en la sociosfera porque así se modifique la tecnosfera será imposible un impacto positivo en la biosfera: "[...] de nada sirven los ajustes tecnológicos sin más, porque lo que debe cambiar es la sociosfera, es decir, el marco de relaciones" (Pardo, 1995, p. 17).

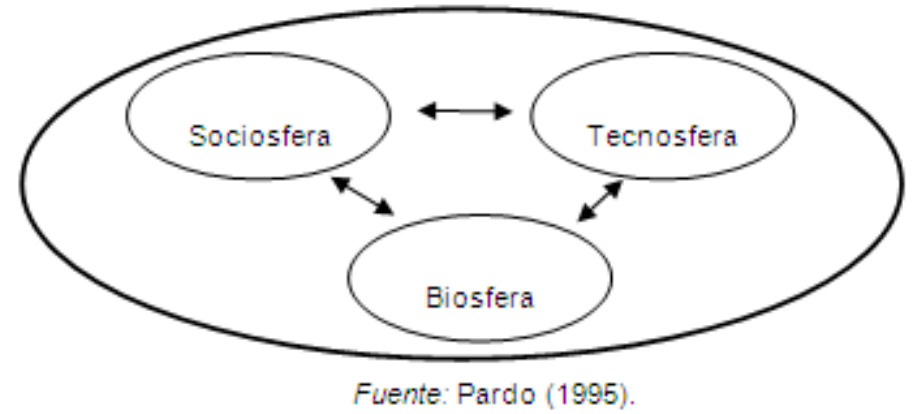

Gráfico 1. Paradigma de la complejidad.

Debido a la influencia del ser humano en el medio que habita, se ha concertado globalmente la EA como una forma de tratar las problemáticas del planeta y por ello ya no se pone en duda la importancia de la EA "como estrategia para concientizar, analizar y comprender la crisis ambiental" (Ministerio de Educación Gobierno de la ciudad de Buenos Aires, 2009, p. 19). Terrón (2000, p. 12) considera que la EA: 
[...] es una dimensión distinta al propósito intrínseco del desarrollo, [que] implica procesos de transmisión y subjetivación donde lo humano es lo prioritario, influida desde luego por la cultura: su ideología y visión de mundo, que derivadas en el plano de los sujetos y de las prácticas sociales, determinan las relaciones que dichos sujetos establecen entre sí y con la naturaleza.

En otras palabras, la EA permite al individuo evaluar analítica y reflexivamente su posición y conducta respecto del medio. La EA es un proceso de formación que busca despertar la conciencia ambiental y que las personas se identifiquen con los graves problemas ambientales (Cugler, 2010) por los que se encuentran pasando las sociedades y el planeta. Desde la perspectiva de Copello, Nunes, Langoni \& Russo (2008, p. 44), la EA "consiste en un modo de ver el mundo, que evidencia las interrelaciones y las interdependencias de los diversos elementos que constituyen y mantienen la vida". Para ello se ha buscado emplear estrategias desde diversos enfoques, las cuales tienen una gran multiplicidad de manifestaciones (Gutiérrez \& Pozo, 2006, p. 22; González \& Valdez, 2012) haciendo de la EA una dimensión compleja (Sauvé, 2003). Sauvé (2004) hace un recorrido de las corrientes de la educación ambiental y que es necesario analizar e interpretar. De acuerdo a la autora, las corrientes para la educación ambiental se han elaborado en dos espacios de tiempo: las primeras corresponden a la década de los setenta y ochenta, y las segundas a necesidades y requerimientos actuales. Las antiguas son: a) corriente naturalista, b) corriente conservacionista/recursista, c) corriente resolutiva, d) corriente sistémica, e) corriente científica, f) corriente humanista y g) corriente moral/ética. Las nuevas son: h) corriente holística, i) corriente bio-regionalista, j) corriente práxica, k) corriente crítica, I) corriente etnográfica, m) corriente de la eco-educación, y n) corriente de la sostenibilidad/sustentabilidad. Las características de cada una de las corrientes se expresan en el Cuadro 2. 
Cuadro 2. Corrientes de la Educación Ambiental

\begin{tabular}{|c|c|}
\hline Corriente & Características \\
\hline \multirow{5}{*}{ Naturalista } & $\begin{array}{l}\text { Concepción del medio ambiente: La naturaleza más allá de los } \\
\text { recursos y del saber que se pueda obtener de ella. }\end{array}$ \\
\hline & $\begin{array}{l}\text { Intención de la EA: Aprender de las cosas de la naturaleza y } \\
\text { aprender a vivir en ella con creatividad. }\end{array}$ \\
\hline & Enfoque privilegiado: Cognitivo. \\
\hline & $\begin{array}{l}\text { Estrategia o modelo pedagógico que ilustra la corriente: } \\
\text { educación al aire libre o natural. }\end{array}$ \\
\hline & $\begin{array}{l}\text { Contexto en el que es pertinente el enfoque: En el contexto en el } \\
\text { que hay ineficacia de la educación ambiental. }\end{array}$ \\
\hline \multirow{5}{*}{ Conservacionista } & Concepción del medio ambiente: La naturaleza como recurso. \\
\hline & $\begin{array}{l}\text { Intención de la EA: Los recursos, tanto en lo que concierne a su } \\
\text { calidad como a su cantidad: el agua, el suelo, la energía, las } \\
\text { plantas (principalmente las plantas comestibles y medicinales) y } \\
\text { los animales (por los recursos que se pueden obtener de ellos), } \\
\text { el patrimonio genético, el patrimonio construido, etc. }\end{array}$ \\
\hline & Enfoque privilegiado: Social. \\
\hline & $\begin{array}{l}\text { Estrategia o modelo pedagógico que ilustra la corriente: } \\
\text { Educación para el consumo y las tres R: Reducción, } \\
\text { Reutilización y Reciclado. }\end{array}$ \\
\hline & $\begin{array}{l}\text { Contexto en el que es pertinente el enfoque: En el contexto } \\
\text { donde hay escasez de los recursos naturales. }\end{array}$ \\
\hline \multirow{5}{*}{ Resolutiva } & $\begin{array}{l}\text { Concepción del medio ambiente: Medio ambiente como conjunto } \\
\text { de problemas. }\end{array}$ \\
\hline & $\begin{array}{l}\text { Intención de la EA: Modificar comportamientos y construir } \\
\text { proyectos colectivos. }\end{array}$ \\
\hline & Enfoque privilegiado: Cognitivo y social. \\
\hline & $\begin{array}{l}\text { Estrategia o modelo pedagógico que ilustra la corriente: } \\
\text { Resolución de problemas, en este caso resolución de } \\
\text { problemáticas ambientales. }\end{array}$ \\
\hline & $\begin{array}{l}\text { Contexto en el que es pertinente el enfoque: En el contexto de } \\
\text { los acelerados y graves problemas ambientales. }\end{array}$ \\
\hline \multirow{5}{*}{ Sistémica } & $\begin{array}{l}\text { Concepción del medio ambiente: Medio ambiente como un } \\
\text { conjunto de componentes interrelacionados tanto biológicos } \\
\text { como sociales. }\end{array}$ \\
\hline & $\begin{array}{l}\text { Intención de la EA: Identificar los componentes del sistema } \\
\text { ambiental y las relaciones de este con el medio social. }\end{array}$ \\
\hline & Enfoque privilegiado: Cognitivo. \\
\hline & $\begin{array}{l}\text { Estrategia o modelo pedagógico que ilustra la corriente: } \\
\text { Acciones al aire libre donde se identifican problemas } \\
\text { ambientales y con base en un proceso interdisciplinar se decide. }\end{array}$ \\
\hline & $\begin{array}{l}\text { Contexto en el que es pertinente el enfoque: En un contexto } \\
\text { donde convergen varios sistemas interconectados y que se } \\
\text { afectan. }\end{array}$ \\
\hline \multirow{5}{*}{ Científica } & $\begin{array}{l}\text { Concepción del medio ambiente: El medio ambiente como un } \\
\text { fenómeno problémico. }\end{array}$ \\
\hline & $\begin{array}{l}\text { Intención de la EA: Abordar con rigor las realidades y } \\
\text { problemáticas ambientales y de comprenderlas mejor, } \\
\text { identificando más específicamente las relaciones de causa a } \\
\text { efecto. }\end{array}$ \\
\hline & Enfoque privilegiado: Cognitivo. \\
\hline & $\begin{array}{l}\text { Estrategia o modelo pedagógico que ilustra la corriente: } \\
\text { Inducción de hipótesis a partir de observaciones y en la } \\
\text { verificación de hipótesis por medio de nuevas observaciones o } \\
\text { por experimentación. }\end{array}$ \\
\hline & $\begin{array}{l}\text { Contexto en el que es pertinente el enfoque: Contextos donde es } \\
\text { necesario el desarrollo de nuevos conocimientos. }\end{array}$ \\
\hline
\end{tabular}




\begin{tabular}{|c|c|}
\hline \multirow{5}{*}{ Humanista } & $\begin{array}{l}\text { Concepción del medio ambiente: Medio ambiente como medio } \\
\text { de vida, con sus dimensiones históricas, culturales, políticas, } \\
\text { económicas, estéticas, etc. }\end{array}$ \\
\hline & $\begin{array}{l}\text { Intención de la EA: Introducir una dimensión humana del medio } \\
\text { ambiente, construido en el cruce entre naturaleza y cultura. }\end{array}$ \\
\hline & Enfoque privilegiado: Cognitivo. \\
\hline & $\begin{array}{l}\text { Estrategia o modelo pedagógico que ilustra la corriente: Explorar } \\
\text { el medio ambiente como medio de vida y construir una } \\
\text { representación de este último: salidas, lecturas, observaciones } \\
\text { dirigidas, contacto con grupos poblacionales. }\end{array}$ \\
\hline & $\begin{array}{l}\text { Contexto en el que es pertinente el enfoque: En donde hay } \\
\text { paisajes suficientes y ricos que permitan el contacto con los } \\
\text { sujetos. }\end{array}$ \\
\hline \multirow{5}{*}{ Moral/Etica } & $\begin{array}{l}\text { Concepción del medio ambiente: El medio ambiente como una } \\
\text { cuestión de orden ético. }\end{array}$ \\
\hline & $\begin{array}{l}\text { Intención de la EA: Formar en valores ambientales, más o } \\
\text { menos conscientes y coherentes entre ellos. }\end{array}$ \\
\hline & Enfoque privilegiado: Afectivo. \\
\hline & $\begin{array}{l}\text { Estrategia o modelo pedagógico que ilustra la corriente: } \\
\text { Desarrollo moral de los alumnos, en vínculo con el desarrollo del } \\
\text { razonamiento socio-científico. Se trata de favorecer la } \\
\text { confrontación en situaciones morales que llevan a hacer sus } \\
\text { propias elecciones y a justificarlas. }\end{array}$ \\
\hline & $\begin{array}{l}\text { Contexto en el que es pertinente el enfoque: Contextos en que la } \\
\text { escala de valores se ha perdido. }\end{array}$ \\
\hline \multirow{5}{*}{ Holística } & $\begin{array}{l}\text { Concepción del medio ambiente: Conjunto de múltiples } \\
\text { dimensiones: realidades socio-ambientales, dimensiones de la } \\
\text { persona que entra en relación con estas realidades, de la } \\
\text { globalidad y de la complejidad de su "ser-en-el-mundo". }\end{array}$ \\
\hline & $\begin{array}{l}\text { Intención de la EA: Formar para comprender la totalidad de cada } \\
\text { ser, de cada realidad y a la red de relaciones que une los seres } \\
\text { entre ellos en conjuntos donde ellos adquieren sentido. }\end{array}$ \\
\hline & Enfoque privilegiado: Cognitivo. \\
\hline & $\begin{array}{l}\text { Estrategia o modelo pedagógico que ilustra la corriente: } \\
\text { Formación en educación ambiental, que integra un enfoque } \\
\text { holístico del aprendizaje y de la relación con el medio, en una } \\
\text { perspectiva psicopedagógica. }\end{array}$ \\
\hline & $\begin{array}{l}\text { Contexto en el que es pertinente el enfoque: Contextos en los } \\
\text { que es posible el desarrollo de procesos de formación. }\end{array}$ \\
\hline \multirow{5}{*}{ Bio-regionalista } & $\begin{array}{l}\text { Concepción del medio ambiente: Medio ambiente desde la } \\
\text { dimensión socio-ecológica (economía y gestión). }\end{array}$ \\
\hline & $\begin{array}{l}\text { Intención de la EA: Formar a las personas para que vuelvan a la } \\
\text { "tierra" mitigando los efectos de la industrialización. }\end{array}$ \\
\hline & Enfoque privilegiado: Social. \\
\hline & $\begin{array}{l}\text { Estrategia o modelo pedagógico que ilustra la corriente: La } \\
\text { educación ambiental está centrada en un enfoque participativo y } \\
\text { comunitario: ella convoca a los padres y a otros miembros de la } \\
\text { comunidad. }\end{array}$ \\
\hline & $\begin{array}{l}\text { Contexto en el que es pertinente el enfoque: En regiones, es } \\
\text { decir, espacios geográficos que comparten un medio natural } \\
\text { homogéneo y una identidad unificada de sus pobladores más } \\
\text { allá de las delimitaciones políticas. }\end{array}$ \\
\hline
\end{tabular}




\begin{tabular}{|c|c|}
\hline \multirow{5}{*}{ Práxica } & $\begin{array}{l}\text { Concepción del medio ambiente: Medio ambiente como ámbito } \\
\text { de cambio a partir de la acción del ser humano. }\end{array}$ \\
\hline & Intención de la EA: Reflexionar y actuar. \\
\hline & Enfoque privilegiado: Social-cognitivo. \\
\hline & $\begin{array}{l}\text { Estrategia o modelo pedagógico que ilustra la corriente: Modelo } \\
\text { investigación-acción. }\end{array}$ \\
\hline & $\begin{array}{l}\text { Contexto en el que es pertinente el enfoque: Contextos en los } \\
\text { que se requiere la participación de varios sujetos para generar } \\
\text { cambios en el medio ambiente. }\end{array}$ \\
\hline \multirow{5}{*}{ Crítica social } & $\begin{array}{l}\text { Concepción del medio ambiente: Medio ambiente influenciado } \\
\text { por las dinámicas sociales. }\end{array}$ \\
\hline & $\begin{array}{l}\text { Intención de la EA: Generar cuestionamientos a los sujetos de } \\
\text { aprendizaje para reconocer las problemáticas del medio y tomar } \\
\text { posturas sobre estas. }\end{array}$ \\
\hline & Enfoque privilegiado: Crítico-social. \\
\hline & $\begin{array}{l}\text { Estrategia o modelo pedagógico que ilustra la corriente: Análisis } \\
\text { de las dinámicas sociales que se encuentran en la base de las } \\
\text { realidades y problemáticas ambientales en } 3 \text { fases: fase crítica, } \\
\text { fase de resistencia y fase de reconstrucción. }\end{array}$ \\
\hline & $\begin{array}{l}\text { Contexto en el que es pertinente el enfoque: Contextos en los } \\
\text { que las problemáticas son altas y el nivel de conciencia y } \\
\text { participación de los sujetos es baja. }\end{array}$ \\
\hline \multirow{5}{*}{ Etnográfica } & $\begin{array}{l}\text { Concepción del medio ambiente: Medio ambiente desde el } \\
\text { carácter cultural de las poblaciones. }\end{array}$ \\
\hline & $\begin{array}{l}\text { Intención de la EA: Integrar un conjunto de culturas para el } \\
\text { desarrollo de la EA. }\end{array}$ \\
\hline & Enfoque privilegiado: Social. \\
\hline & $\begin{array}{l}\text { Estrategia o modelo pedagógico que ilustra la corriente: Adaptar } \\
\text { la pedagogía a las realidades culturales diferentes, inspirándose } \\
\text { en estas: lengua, costumbres, tradiciones, leyendas, etc. }\end{array}$ \\
\hline & $\begin{array}{l}\text { Contexto en el que es pertinente el enfoque: Contextos en los } \\
\text { que se posibilita el contacto con grupos étnicos. }\end{array}$ \\
\hline \multirow{5}{*}{ Eco-educación } & $\begin{array}{l}\text { Concepción del medio ambiente: Esfera de interacción esencial } \\
\text { para la eco-formación y la eco-ontogénesis. }\end{array}$ \\
\hline & $\begin{array}{l}\text { Intención de la EA: Posibilitar el desarrollo personal del sujeto a } \\
\text { partir del contacto con el medio ambiente. }\end{array}$ \\
\hline & Enfoque privilegiado: Social. \\
\hline & $\begin{array}{l}\text { Estrategia o modelo pedagógico que ilustra la corriente: } \\
\text { Caracterizar y diferenciar los períodos particulares en lo relativo } \\
\text { a los tipos de relación con el medio ambiente y asociar a ello } \\
\text { prácticas específicas de educación ambiental. }\end{array}$ \\
\hline & Contexto en el que es pertinente el enfoque: Cualquier contexto. \\
\hline \multirow{5}{*}{ Sostenibilidad } & $\begin{array}{l}\text { Concepción del medio ambiente: Medio ambiente desde el } \\
\text { enfoque económico. }\end{array}$ \\
\hline & $\begin{array}{l}\text { Intención de la EA: Adecuar la EA al Programa de Educación } \\
\text { para un futuro viable de la UNESCO (1997) el cual tiene su } \\
\text { origen en la Cumbre de la Tierra de 1992. Esto es el cuidado y } \\
\text { distribución de los recursos de tal suerte que no afecte las } \\
\text { futuras generaciones. }\end{array}$ \\
\hline & Enfoque privilegiado: Naturalista. \\
\hline & $\begin{array}{l}\text { Estrategia o modelo pedagógico que ilustra la corriente: } \\
\text { Proporcionar la información sobre los productos (modos de } \\
\text { producción, posibles impactos ambientales, costos de } \\
\text { publicidad, etc.) y desarrollar en los consumidores capacidades } \\
\text { de elección entre diferentes opciones. }\end{array}$ \\
\hline & Contexto en el que es pertinente el enfoque: Cualquier contexto. \\
\hline
\end{tabular}

Fuente: Elaboración Drodia a Dartir de Sauvé (2004).

Sauvé (2004) hace un desarrollo importante de las corrientes de la EA y cabe destacar que no hay un criterio homogéneo para la gestión de estos procesos. Es necesario conocer el grupo de sujetos, su contexto, intereses y necesidades, así como el tipo de sociedad. La aplicación de un modelo de EA depende de las circunstancias propias en las que se encuentran inmersos los sujetos de 
aprendizaje, es decir, a los contextos de los educandos y al modelo de sociedad del momento. En el Cuadro 3 se exponen las características vitales de cada década en materia ecológica y ambiental desde los años sesenta hasta los noventa a fin de establecer las relaciones entre las corrientes que se han desarrollado y las cualidades propias de cada sociedad. Si bien los años sesenta fue el periodo inicial en la construcción de nuevas perspectivas en muchos campos de la vida del hombre, solo hasta los años setenta se da una preocupación real y relevante por los asuntos ambientales. Es precisamente en este periodo que la educación construye modelos de enseñanza como los expuestos por Sauvé (2004). Por ello, las primeras corrientes se fundamentaron en asociar los tópicos medioambientales con problemáticas y recursos naturales, precisamente cuando se evidenció la escasez de los segundos y los efectos de los primeros. Posteriormente, las corrientes incluirán aspectos económicos y políticos, precisamente porque los años ochenta y noventa correspondieron a cambios de ese tipo.

Cuadro 3. Características relevantes en materia ecológica y ambiental por periodos (sesenta-noventa)

\begin{tabular}{|c|c|}
\hline Década & Características \\
\hline Los sesenta & $\begin{array}{l}\text { Los años sesenta correspondieron a un periodo de muchos cambios } \\
\text { y entre estos está el inicio de una preocupación ambiental basada en } \\
\text { hechos como la creciente desigualdad social, la crisis petrolera y la } \\
\text { contaminación ambiental. Este último tema, pasó a integrar las } \\
\text { agendas de las reuniones de grupos ambientalistas, las denuncias se } \\
\text { hicieron presentes y los informes científicos sobre el agotamiento de } \\
\text { los recursos marcaron el inicio del llamado a la conciencia ecológica. }\end{array}$ \\
\hline Los setenta & $\begin{array}{l}\text { Los temas sobre la crisis del petróleo así como el crecimiento } \\
\text { demográfico fueron temas ambientales de las agendas científicas y } \\
\text { políticas. Los resultados del proceso iniciado en los sesenta se } \\
\text { multiplicaron en esta década. La crisis del petróleo condujo al } \\
\text { desarrollo de nuevas energías como la nuclear, la cual fue motivo de } \\
\text { protesta por grupos ambientalistas. } \\
\text { Como ya se comprendía que los recursos no eran suficientes por el } \\
\text { crecimiento demográfico, se expusieron soluciones como el control de } \\
\text { natalidad. } \\
\text { Del mismo modo, la pobreza y la hambruna en regiones como el } \\
\text { Africa, demandaron toma de decisiones de tipo ambiental y políticas. }\end{array}$ \\
\hline Los ochenta & $\begin{array}{l}\text { En este periodo se da una importante mirada a los asuntos } \\
\text { ambientales ya que son incluidos en las políticas de los gobiernos } \\
\text { dentro de los Estados. Se convierten las cuestiones } \\
\text { medioambientales en parte de los temas de la geopolítica mundial. } \\
\text { Los recursos naturales han sido explotados en exceso por las } \\
\text { potencias industrializadas y el deterioro del ambiente es evidente. Del } \\
\text { mismo modo, catástrofes producidas por accidentes en empresas } \\
\text { ponen sobre la mesa riesgos latentes que afectan la naturaleza y al } \\
\text { hombre. Aparece el concepto de desarrollo sustentable. }\end{array}$ \\
\hline Los noventa & $\begin{array}{l}\text { Desde el comienzo de la década de los noventa se dieron } \\
\text { importantes cambios, especialmente, en la acción pública de los } \\
\text { Estados quienes se comprometieron a través de acuerdos } \\
\text { internacionales. Por ejemplo, el Tratado de Río de Janeiro de 1992, } \\
\text { producto de la Conferencia de las Naciones Unidas para el Medio } \\
\text { Ambiente y el Desarrollo. Con este tratado se hace concreta la } \\
\text { responsabilidad de los Estados con el desarrollo sostenible. }\end{array}$ \\
\hline
\end{tabular}

El medioambiente como objeto de estudio desde las ciencias de la educación es un tema relativamente nuevo. Sin embargo, frente al auge del tema medioambiental se han logrado diseñar variados enfoques para la EA lo cual permite, desde el punto de vista teórico, el uso de diversas estrategias atendiendo a las necesidades de las comunidades y a las características de los educandos. De igual manera, se deben atender de manera prioritaria los asuntos éticos de la EA así como su fundamentación ciudadana. 
Terrón (2000, p. 12) ha dicho que formar a los educandos en términos de EA "significa ayudarles a comprender cuál es el sustrato ético que los orienta, el por qué y el para qué de sus acciones, la importancia de estar conscientes de [ellos] mismos, de [su] persona y del objetivo que [se persigue] en la vida", en otras palabras, de dar un sentido al hombre dentro del cosmos. De allí también se destaca un elemento importante que impregna la noción de formación en la actualidad: los procesos metacognitivos. Esto es así, porque un proceso de formación que atiende a los principios de la metacognición promueve la capacidad que tiene el sujeto para aprender por sí solo. Para Casado (1998, p. 100) la metacognición hace:

[...] referencia a la reflexión que el sujeto de aprendizaje realiza sobre su manera de aprender, su estilo, y las consecuencias que obtiene para posteriores aprendizajes. El resultado de esta reflexión, por tanto, debería ser la adopción de estrategias eficaces ante un problema, en lugar del acercamiento mediante un sistemático ensayo-error hasta que llegue el éxito.

Avendaño \& Parada (2011, p. 408) especifican que la metacognición es un requisito sine qua non, dentro de los actuales modelos pedagógicos, y que con ella "se logra la autorregulación del organismo, lo que conlleva al aprendizaje autónomo". Desde el punto de vista de la EA, los procesos de formación enfocados en la metacognición posibilitan que los sujetos de aprendizaje asuman roles realmente trascendentales en el hábitat donde se desenvuelven logrando identificar los efectos de cada acción que realizan. Y esto es coherente con la idea de que la educación en ciencias no puede soslayar el papel del individuo y su contexto, a fin de que los desafíos de la educación no se dan en un vacío, formando parte de la compleja maraña de la dinámica económica, social, cultural y política de la época (Stavenhagen, 2000). Los apuntes de Stavenhagen (2000) incluyen parte de las viejas y nuevas corrientes de las que expresa Sauvé (2004) y es esencial reconocer y analizar contextos de educandos así como su rol, características del entorno, necesidades del medio, normatividad y políticas públicas.

Los contenidos se convierten en medios para lograr habilidades y competencias de tipo cognitivo (Avendaño \& Parada, 2011, p. 408). Así, la educación ambiental no debe estar basada en la transmisión de los contenidos específicos, ya que no existe un contenido único, siendo, por el contrario, un conjunto de temas ampliamente variados, y que se utilizan dependiendo de los niveles a que se destinan y de los contextos educativos en los que se procesan las actividades (Reigota, 1995, p. 70).

La naturaleza de la EA implica para el sujeto un avance no solo en lo cognitivo sino también en la esencia que brinda un enfoque crítico como lo estima Delors (1996, p. 45). Freire (1992a, p. 24) afirma que los hombres se encuentran a sí mismos como un problema, y dicha autoperspectiva del hombre está ligada a su puesto en el cosmos y a su interacción con él. En EA esto resulta muy importante, pues las relaciones del hombre con el medio, el cosmos o su entorno resultan ser un problema que debe ser reflexionado para conducir a un estado más humano, hecho que lo asume Freire (1992a, p. 24) en el siguiente sentido: "el problema de su humanización, a pesar de haber sido siempre, desde un punto de vista axiológico, su problema central, asume hoy el carácter de preocupación ineludible". Y como afirma este mismo autor, el reconocimiento de la necesidad de llevar a cabo procesos de humanización significa que hay una conciencia en el hombre de su propia deshumanización. En dicho sentido, podríamos decir que la EA encuentra su esencia en el mismo hombre, cuya humanización se encuentra inconclusa y de la cual él es consciente. 
Para Freire (1992b, p. 7) la educación es "praxis, reflexión y acción del hombre sobre el mundo para transformarlo", y dicha noción se puede extrapolar a las pretensiones de la EA. Por otro lado, la noción política de la educación (Monroy, 2005) nos recuerda que la "educación es un verdadero producto cultural, social e histórico" (Avendaño \& Parada, 2011, p. 400). En esta misma línea lo considera Díaz (2004) para quien los problemas ambientales y su comprensión son cuestiones socio-políticas y el proceso de edificación del conocimiento como forma de concientización ambiental, constituye quehaceres complejos y necesarios. La UNESCO (1994, p. 20) describe que "[...] cualquier disciplina precisa de otras disciplinas afines [...] [y] tratándose de problemas del medio ambiente; un mismo fenómeno debe ser estudiado bajo diferentes enfoques, aunque complementarios".

En EA el papel de la conciencia tiene un rol vital. Triviños (1987, p. 37), considera que:

[...] la conciencia presupone una reflexión sobre la realidad objetiva y por lo tanto, la formación de ciudadanos conscientes exige una metodología educativa centrada en la problematización de las situaciones, donde cada uno de los involucrados en el proceso puedan interiorizar valores y establecer juicios.

De esta manera, se estarían formando hombres con un rol positivo dentro de la sociedad que pueden asumir comportamientos que benefician el medio ambiente, pero desde una construcción cultural que permite su solidificación y permanencia en los demás individuos actuales y futuros. La comprensión de la relación que se establece entre el individuo y su entorno es fundamental para entender la relación hombre-medio, con el objetivo de generar una conciencia crítica-reflexiva capaz de construir soluciones y alternativas frente a los problemas ambientales emergentes a partir de acciones educacionales comprometidas con la mejora de la calidad de vida para la colectividad (Castelltort, 2007, p. 368).

Para un efectivo proceso de EA, y en el marco de generar conciencia, resignificar la cultura y el desarrollo, es necesario que los maestros tengan una compleja formación ya que es necesaria en las transformaciones (Nóvoa, 2009, p. 210) que se quieran adelantar. Ello exige, por parte de los maestros, una completa compresión de las problemáticas ambientales y la enumeración de posibles soluciones que abarque diversos factores. La EA constituye un proceso no solo de tipo epistémico, ético o cosmológico. Como se mencionó, es un elemento integral de la política actual que permite la consolidación de valores supremos como la igualdad, la fraternidad, la libertad, la justicia y la dignidad. Para la Organización de las Naciones Unidas para la Educación, la Ciencia y la Cultura (UNESCO) en los "numerosos desafíos del porvenir, la educación constituye un instrumento indispensable para que la humanidad pueda progresar hacia los ideales de paz, libertad y justicia social" (Delors, 1996, p. 7).

De la mano de esta apreciación se puede enaltecer la esencia de la educación en el desarrollo continuo de la persona y las sociedades, siendo este un mecanismo para alcanzar un desarrollo humano más armonioso, más genuino; para hacer retroceder la pobreza, la exclusión, las incomprensiones, las opresiones, las guerras, y todo aquello que resulta ser un compendio de una misma problemática. Los hombres en sus construcciones sociales e invenciones solo han encontrado un instrumento para la consolidación de la humanidad y ha sido la educación. Ella permitirá, en la medida en que sea modificada, alcanzar transformaciones significativas en todos los campos y de ahí que la apuesta para darle un nuevo significado al medio ambiente dependa, esencialmente, del 
sentido y el papel que se le imprima a la educación. La educación debe ser observada como una herramienta fundamental en la formación de ciudadanos amigables con el medio ambiente y la perspectiva resaltada de la EA incluye elementos significativos como la paz, la violencia, la democracia y la pobreza, los cuales son fundamentales desde el punto de vista pedagógico. Su fundamento ya se encuentra en el pensamiento crítico de Freire o en el enfoque cognitivo de Gardner $(2008$, p. 75,89$)$ para quien es vital en la actualidad y en el futuro desarrollar cinco tipos de mentes, entre esas, la mente respetuosa y la mente ética, las cuales permiten construcciones sociales basadas en el respeto al otro y la unificación de valores que propenden por ambientes más sanos. En este sentido, la EA debe dirigirse hacia la comprensión de la realidad del sujeto y al despertar de la conciencia del mismo que le permita identificarse como ser posibilitador de cambios.

\section{REFERENCIAS}

- Acuña, G. (2010). El acceso a la información y participación pública en la toma de decisiones ambientales en América Latina: avances y desafíos hacia una mejor aplicación del Principio 10 de la Declaración de Río. En Organización de las Naciones Unidas (ONU) \& PNUMA. Quinto Programa Regional de Capacitación en Derecho y Políticas Ambientales. Panamá: ONU y PNUMA.

- Avendaño, W. (2011). Responsabilidad Social Corporativa (RSC) y desarrollo sostenible: una mirada desde la Declaración de Río de 1992. Respuestas, 16(2), 45-59.

- Avendaño, W. \& Parada, A. (2011). Un modelo pedagógico para la reproducción y transformación cultural en las sociedades del conocimiento. Investigación y Desarrollo, 19(2), 398-413.

- Ball, S. (Comp.). (2001). Foucault y la educación. Disciplinas y saberes. Madrid: Morata.

- Brañes, R. (2001). Informe sobre el desarrollo ambiental latinoamericano: su aplicación después de diez años de la conferencia de las Naciones Unidas sobre el Medio Ambiente y el Desarrollo. México: PNUMA.

- Casado, M. (1998). Metacognición y motivación en el aula. Revista de Psicodidáctica, 6, 99-107.

- Casares, J. (2000). Acciones para el desarrollo de la educación ambiental en Galicia. En UNESCO \& Junta de Galicia. Reunión Internacional de Expertos en Educación Ambiental: Nuevas Propuestas para la Acción (Actas). Santiago de Compostela: Litonor.

- Castelltort, A. (2007). Elaboración de un instrumento para orientar el diseño y evaluar propuestas educativas en el campo de la educación ambiental. En Pujol, R. \& Cano, L. (Eds.). Nuevas tendencias en investigaciones en Educación Ambiental (pp. 365-383). Madrid: Organismo Autónomo Parques Nacionales / Ministerio de Medio Ambiente.

- Carta de Belgrado. Seminario de Educación Ambiental, 13-22 de octubre de 1975.

- Colombia, Congreso de la República. Ley 115 de 1994 (Ley General de Educación).

- Colombia, Congreso de la República. Constitución Política de 1991.

- Copello, M., Nunes, M., Langoni, M. \& Russo, D. (2008). Algunas propuestas de educación ambiental en la escuela. Parte II: Uso responsable del agua: ¿Es un problema? ¿Yo tengo algo que ver con él? Quehacer Educativo, abril, 44-51. 
- Cugler, A. (2010). ¿Se puede aplicar la educación ambiental en la actividad turística? Ponencia presentada en la Conferencia Turismo y Educación Ambiental, julio, Lima, Perú.

- Cumbre Mundial sobre el Desarrollo Sostenible (2002), 26 de agosto al 4 de septiembre, Johannesburgo, Sudáfrica.

- Declaración de Río sobre el Medio Ambiente y el Desarrollo, 14 de junio de 1992, Río de Janeiro, Brasil.

- Declaración Salónica, 8-12 de diciembre de 1997, Salónica, Grecia.

- Delors, J. (1996). La educación encierra un tesoro. Informe a la UNESCO de la Comisión Internacional sobre la educación para el siglo XXI. Madrid: Santillana.

- Dewey, J. (2004). Democracia y educación. Madrid: Morata.

- Díaz, M. (2004). Análisis de las estrategias de Educación Ambiental autonómicas. Tendencias y perspectivas. Proyecto de suficiencia investigadores del Programa Interuniversitario de Educación Ambiental. Madrid: Universidad Autónoma de Madrid.

- Durkheim, E. (1976). La educación como socialización. Salamanca: Sígueme.

- Fernández, R. \& Leiva, M. (2003). Ecología para la agricultura. Madrid: Mundi-Prensa.

- Fernández, M., Moreno, L., De la Losa, A. \& Calaforra, J. (2005). Propuesta de un índice de impacto sobre el medio hídrico subterráneo del empleo del suelo como elemento activo de la depuración de aguas residuales urbanas. En López, J., Rubio, J. \& Martín, M. (Eds.). VI Simposio del Agua en Andalucía (Tomo II) (pp. 1057-1068). Madrid: Instituto Geológico y Minero de España.

- Freire, P. (1992a). Pedagogía del oprimido. Madrid: Siglo XXI.

- _ (1992b). La educación como práctica de la libertad. Madrid: $\overline{\text { Siglo XXI. }}$

- Gardner, H. (2008). Las cinco mentes del Futuro. Bogotá: Paidós

- Giroux, H. (1986). Teorías de la reproducción y la resistencia en la nueva sociología de la educación: un análisis crítico. Revista Colombiana de Educación, 17, 61-108.

- Gómez, D. (2002). Evaluación de impacto ambiental. Madrid: MundiPrensa.

- González, E. \& Valdez, R. (2012). Enfoques y sujetos en los estudios sobre representaciones sociales de medio ambiente en tres países de Iberoamérica. Revista de Investigación Educativa, 14. Recuperado de http://www.uv.mx/cpue/num14/inves/gonzalez_valdez medio_ambiente.html

- Gutiérrez, J. \& Pozo, T. (2006). Modelos teóricos contemporáneos y marcos de fundamentación de la educación ambiental para el desarrollo sostenible. Revista Iberoamericana de Educación, 41, 21-68.

- Leff, E. (2000). Globalización y complejidad ambiental. En UNESCO \& Junta de Galicia. Reunión Internacional de Expertos en Educación Ambiental: Nuevas Propuestas para la Acción (Actas) (pp. 67-97). Santiago de Compostela: Litonor.

- Martínez, V. (2005). ESO 4. Barcelona: Paidotribo.

- Ministerio de Educación - Gobierno de la ciudad de Buenos Aires. (2009). Educación ambiental: de la conservación a la formación para la ciudadanía. Buenos Aires: Ministerio de Educación.

- Ministerio de Educación Nacional de Colombia. (1995). Serie Lineamientos Curriculares para el área de Ciencias Naturales y Educación Ambiental. Bogotá: Ministerio de Educación Nacional.

- Ministerio del Medio Ambiente \& Ministerio de Educación Nacional de Colombia. (2002). Política de Educación Ambiental SINA. Bogotá: 
Ministerio del Medio Ambiente y Ministerio de Educación Nacional. Recuperado de http://www.humboldt.org.coliavh/documentos/politica/ politicas_ambientales/2002\%20Politica\%20Nacional\%20de\% 20Educacion\%20Ambiental.pdf

- Monroy, B. (2005). Pedagogía cognitiva en las sociedades de la información. Documento de trabajo. Bogotá: Universidad Externado de Colombia.

- Moreno, F. (2008). Origen, concepto y evolución de la Educación Ambiental. Innovación y Experiencias Educativas, 13. Recuperado de http://www.csi-csif.es/andalucia/modules/mod_ense/revista/ pdf/Numero_13/FRANCISCO_MORENO_1.pdf

- Nóvoa, A. (2009). Para una formación de profesores construida dentro de la profesión. Revista de Educación, 350, 203-218.

- Orellana, I. (2002). La estrategia pedagógica de la comunidad de aprendizaje: definiendo sus fundamentos, sus prácticas y su pertinencia en educación ambiental. En Sauvé, L., Orellana, I. \& Sato, M (Comps.). Textos escogidos en educación ambiental de una América a otra (pp. 221232). Québec: ERE-UQAM-EDAMZ.

- Organización de las Naciones Unidas (ONU). (1972). Declaración de Estocolmo sobre el Medio Ambiente Humano. Estocolmo, 5-16 de junio.

- Organización de las Naciones Unidas para la Educación, la Ciencia y la Cultura (UNESCO) \& Programa de Naciones Unidas para el Medio Ambiente (PNUMA). (1977). Conferencia Intergubernamental sobre Educación relativa al Medio Ambiente. Tbilisi (URSS), 14-26 de octubre.

- . (1987). Congreso Internacional de Educación y Formación sobre Medio Ambiente. Moscú (URSS), 17-21 de agosto.

- Organización de las Naciones Unidas para la Educación, la Ciencia y la Cultura (UNESCO). (1994). Enfoque interdisciplinar en la educación ambiental. Bilbao: Los libros de la Catarata.

- $\quad$ (1998). Hacia una educación sin exclusiones. Santiago de Chile: Oficina Regional de Educación de la UNESCO.

- $\quad$ (1998b).

- Pardo, A. (1995). La educación ambiental como proyecto. Barcelona: Horsori.

- Pérez, G. (2003). Pedagogía Social, Educación Social: Construcción Científica e Intervención Práctica. Madrid: Narcea.

- Pilonieta, G. (2010). Modificabilidad estructural cognitiva y educación. Bogotá: Magisterio.

- Porto, C. (2000). Las múltiples y contradictorias prácticas de la educación ambiental. Formación Ambiental, 12(26), 11-13.

- Reigota, M. (1995). Meio ambiente e representação social. São Paulo: Cortez.

- Sauvé, L. (2003). Perspectivas curriculares para la formación de formadores en educación ambiental. Ponencia en el I Foro Nacional sobre la Incorporación de la Perspectiva Ambiental en la Formación Técnica y Profesional, Universidad Autónoma de San Luis de Potosí (México), 9-13 de junio.

- (2004). Una cartografía de corrientes en educación ambiental. En Sato, M. \& Carvalho, I. (Orgs.). A pesquisa em educação ambiental: cartografias de uma identidade narrativa em formação. Porto Alegre: Artmed.

- Stavenhagen, R. (2000). Tendencias del debate educativo a nivel mundial: El Informe de la Comisión Internacional sobre la Educación para el Siglo XXI. Ponencia del VIII Simposio de Educación Cátedra Paulo Freire, ITESO, 23-26 febrero, Guadalajara, México. 
- Terrón, E. (2000). La educación ambiental ante los desafíos del siglo XXI. Revista de la Academia Mexicana de Profesores de Ciencias Naturales A. C., (3), 5-13.

- Triviños, A. (1987). Introdução à pesquisa em ciências sociais: a pesquisa qualitativa em educação. São Paulo: Atlas.

- Tyler, R. (1986). Principios básicos del currículo. Buenos Aires: Troquel.

- Valdés, O. (2000). La educación ambiental y protección de la biodiversidad de proyectos y programas en Cuba. En UNESCO \& Junta de Galicia. Reunión Internacional de Expertos en Educación Ambiental: Nuevas Propuestas para la Acción (Actas) (pp. 623-644). Santiago de Compostela: Litonor.

- Vázquez, F. A. (1975). Notas sobre el sistema jurídico mexicano, a la luz de la Constitución. Jurídica, Anuario, 7, 587-641.

1. Artículo de revisión.

2. Administrador de Empresas. Especialista en Gerencia Educativa. Magíster en Gerencia de Empresas. Doctorando en Educación. Docente, Universidad Francisco de Paula Santander. Investigador, Grupo de Investigación "GICSH" en Ciencias Sociales y Humanas, adscrito a la Universidad Francisco de Paula Santander. Ipazmontes@gmail.com

3. Economista. Especialista en Alta Gerencia. Magíster en Administración. Doctorando en Ciencias Sociales y Humanas. Docente-Investigador, Universidad Francisco de Paula Santander (Cúcuta), Director Grupo de Investigación "GICSH" en Ciencias Sociales y Humanas. wrac2008@hotmail.com

4. Abogado. Especialista en Alta Gerencia. Magíster en Educación. DirectivoDocente del Colegio Militar General Francisco de Paula Santander (Cúcuta). Investigador, Grupo de Investigación "GICSH" en Ciencias Sociales y Humanas, adscrito a la Universidad Francisco de Paula Santander. abadernesto@hotmail.com

5. Esta Conferencia ofrecerá las bases para determinar los objetivos y logros básicos de la EA en Colombia a través de los Lineamientos Curriculares para el área de Ciencias Naturales y educación Ambiental (1995).

Para citar este artículo: Paz-M., L.S., Avendaño-C, W.R., Parada-Trujillo, A. (2014). Desarrollo conceptual de la educación ambiental en el contexto colombiano. Revista Luna Azul, 39, 250-270. Recuperado de http://lunazul.ucaldas.edu.co/index.php?option=content\&task=view\&id=958 